\title{
Image Digitising and Analysis of Outflows from Young Stars
}

\author{
W. J. Zealey and S. L. Mader \\ Department of Physics, University of Wollongong, Northfield Avenue, \\ Wollongong, NSW 2522, Australia. \\ b.zealey@uow.edu.au \\ Received 1996 September 14, accepted 1997 March 27
}

\begin{abstract}
We present IIIaJ, IIIaF and IVN band images of HH objects digitised from the ESO/SERC Southern Sky Survey plates. These form part of a digital image database of southern $\mathrm{HH}$ objects, which allows the identification of emission and reflection nebulosity and the location of the obscured sources of outflows.
\end{abstract}

Keywords: image processing - ISM: jets and outflows - ISM: individual objects: (HH 34, HH 46/47) — stars: formation

\section{Introduction}

The availability of the ESO/SERC Sky Survey has made scanning possible of the southern skies for planetary nebulae, supernova remnants and diffuse nebulae. Several catalogues of sources on these plates have been published, sometimes accompanied by photographs of the more important objects.

With the advent of low-cost PC computers, frame grabbers and video cameras it has become possible to digitise and enhance areas of interest on these survey plates. Applying digital techniques to the images from the IIIaJ, IIIaF and IVN surveys allows the separation of line and continuum sources in the galactic plane (Zealey et al. 1994).

In this paper we outline an application of digital methods to the study of outflows driven by mass loss from young stellar objects (YSOs). Evidence that a YSO is undergoing mass loss is provided through the effect the stellar wind has on the surrounding medium. Phenomena such as bipolar molecular outflows, masers and the Herbig-Haro (HH) objects and jets are all indicators of a YSO undergoing mass loss.

Observations at optical and infrared wavelengths have shown that $\mathrm{HH}$ objects and jets are regions of shock-excited gas emitting in $\mathrm{H} \alpha$ and forbidden excitation lines of $[\mathrm{O}$ I] $\lambda \lambda 6300,6363$ and [S II] $\lambda \lambda 6716,6731$ in the visible and $\mathrm{H}_{2}(2 \cdot 12 \mu \mathrm{m})$ in the infrared. The different shock structures and excitation conditions of a $\mathrm{HH}$ complex can be distinguished by comparing images obtained in optical and infrared emission lines with differing excitation parameters, e.g. [S II] emission is indicative of $v_{\text {shock }} \approx 100 \mathrm{~km} \mathrm{~s}^{-1}$, while the presence of $\mathrm{H}_{2}$ emission indicates slower shocks $\left(v_{\text {shock }} \approx 40 \mathrm{~km} \mathrm{~s}^{-1}\right)$.

$\mathrm{HH}$ objects and jets are associated with both low-luminosity $\left(L_{\text {bol }} \approx 1-100 L_{\odot}\right) \mathrm{T}$ Tauri stars and high-luminosity YSOs $\left(L_{\text {bol }} \approx 10^{3} L_{\odot}\right)$ such as IRAS sources (Mundt 1988; Reipurth 1994). The visibility of the source depends on the amount of obscuration, the orientation of the flow and circumstellar disk. Due to heavy extinction from the surrounding star forming clouds, many YSOs are obscured at optical wavelengths. Near-infrared images often provide the first identification of the driving source of the outflow as the optical extinction becomes less important at longer wavelengths.

Morphological features present in most outflows include:

- an embedded YSO which drives the outflow;

- a collimated jet and counter jet;

- a section where the jet disappears, but isolated emission knots remain;

- a working surface (bow shock) with associated $\mathrm{HH}$ complex.

\section{Optical Surveys and Morphological Studies}

The abundance of nearby stellar outflow complexes accessible to observation from the southern hemisphere provides us with the opportunity to study highly supersonic outflows for comparison with theoretical models. Since the publication of the original catalogue of $43 \mathrm{HH}$ objects (Herbig 1972), there has been a substantial increase in the number of known sources due to advances in imaging technology, especially in CCD imagery through narrow-band filters. Catalogues have been compiled by Schwartz (1977; deep red objective-prism survey of southern $\mathrm{HH}$ objects), Hartigan \& Lada (1985; V, R, I and H $\alpha$ CCD images of suspected HH objects), Strom et al. (1986; broadband V, I, H $\alpha$ and [SII] CCD images), Von Hippel, Burnell \& Williams (1988; literature survey up to 1988) and Reipurth (1994; extensive notes on individual $\mathrm{HH}$ objects with detailed references to the literature). Plans are under way to incorporate [S II], $\mathrm{H} \alpha$ and broadband images into Reipurth's catalogue (Reipurth 1995, private communications). 
The ESO/SERC surveys provide matching deep IIIaJ, IIIaF and IVN images of the southern sky. Although Reipurth and others have searched the survey material for $\mathrm{HH}$ objects, little systematic use has been made of them to identify the driving sources of these flows. $\mathrm{HH}$ objects lying close to bright reflection nebula are often swamped by reflected light on IIIaJ and IIIaF plates. Digital imaging allows sophisticated enhancement techniques to be applied to areas of interest. Use of photographic and, more recently, digital unsharp masking techniques allows the detection of new emission sources e.g. HH 247 (Malin, Ogura \& Walsh 1987). It is also possible to overlay IIIaJ, IIIaF and IVN images with infrared and radio maps of sources.

\section{Video Digitising Systems}

We are digitally imaging all known southern stellar HH complexes selected from Reipurth's (1994) catalogue of $\mathrm{HH}$ objects using a low-cost $\mathrm{PC} / \mathrm{AT}$ video digitising system. This system has been developed at the Department of Physics of the University of Wollongong from commercially available hardware and software.

The image digitising facility provides for the real-time, 8-bit (256 grey-scale) video digitising and analysis of photographic plate material (Zealey et al. 1994). The image digitiser system uses a PC/AT based video frame grabber.

The digitising board used is of PCVisionPlus type. Computer controlled look up tables (LUTs) allow the stored $512 \times 512$ pixel image to be displayed in pseudo-colour on a dedicated RGB image monitor. The video digitiser accepts video input from a Philips CCD video camera mounted on a Polaroid Laboratory Camera; a combination of lenses allows for both wide-field ( $300 \mathrm{~mm}$ square; $6 \mathrm{~mm}$ pixels) and microscopic ( $5 \mathrm{~mm}$ square; $10 \mu \mathrm{m}$ pixels) imaging.

Plate illumination is provided either by a Chromega colour enlarger head for regions up to $5 \mathrm{~cm}$ square or a light box for larger areas.

Jandell's Java software allows real-time enhancement and measurement of the digitised images. Java allows for spatial filtering, contrast enhancement, thresholding, simple backgrounding and source counting, as well as photometric analysis. Images are stored as 8-bit TIFF (tagged image file format) files which are readable by a wide range of image software. Once images are captured from the screen, they are stored on a $600 \mathrm{Mb}$ Ricoh magneto optical drive and then copied onto floppy disks for transfer to other PCs with specialised imaging software. Image editing is done with JPL's Alchemy Mindworks GWS package, which is useful for a first look at the images, cropping and scaling and conversion to GIF (graphic interchange format) or other image formats. Further enhancement and addition of text is done in CorelDraw or Adobe Photoshop.

\section{Examples of Digital Images}

At present there are over $250 \mathrm{HH}$ objects listed in Reipurth's (1994) catalogue of $\mathrm{HH}$ objects. The digital images obtained from the ESO/SERC IIIaJ, IIIaF and IVN survey material can be used to discriminate between emission and reflection nebulosity and to identify heavily reddened continuum sources.

The IIIaJ and IIIaF images provide a roadmap of shock-excited gas. The IIIaJ + GG395 plate/filter combination ( $3950 \AA \leq \lambda \leq 5400 \AA$ ) is responsive to high-excitation emission lines which include the [O III] $\lambda \lambda 4959,5007$ line.

The IIIaF + RG630 plate/filter combination $(6300 \AA \leq \lambda \leq 6900 \AA)$ is dominated by the lowexcitation $\mathrm{H} \alpha,\left[\mathrm{N}_{\mathrm{II}}\right] \lambda \lambda 6548,6583$ and [S II] $\lambda \lambda 6717,6731$ emission lines.

The IVN plates $(6950 \AA \leq \lambda \leq 9000 \AA)$ do not contain any major emission lines that are known to be prominent in shock-excited regions of $\mathrm{HH}$ objects. The IVN band images therefore act as a probe for scattered continuum light from the embedded YSO.

The IIIaJ and IIIaF images can used together with published $\mathrm{H}_{2}$ infrared images to clearly identify shock-excited emission regions over a range of excitation conditions (Zealey et al. 1992). In many cases, emission features in the IIIaJ, IIIaF and $\mathrm{H}_{2}$ images do not coincide, which indicates that either obscuration or excitation conditions vary across the objects.

The IVN images can be combined with published near-infrared and continuum images to identify the driving sources of the outflows.

Examples of the digitised images are presented in Figures 1 and 2 for the $\mathrm{HH} 34$ and $\mathrm{HH}$ 46/47 systems respectively. Both areas have also been studied in detail using CCD and IR cameras allowing a clear comparison to be made of the usefulness of the IIIaJ, IIIaF and IVN band digital imaging.

\section{$4.1 \mathrm{HH} 34$}

HH 34 is an archetypal outflow which has been well studied at visible, infrared and millimetre wavelengths. Inspection of the digitised IIIaJ and $\mathrm{IIIaF}$ images (Figure 1) shows similar features to those visible in the deep [S II] image of Mundt (1988). Visible are the bow-shaped emission nebulosity $\mathrm{HH}$ $34 \mathrm{~S}$ and the continuum reflection nebula Re 24 (Reipurth 1985).

The IVN image (Figure 1) shows many of the strong infrared sources, including HH 34 IRS, IRS5 and $\operatorname{Re} 22$ (Reipurth 1985).

The reflection nebulosity Re 23 (Reipurth 1985) may represent the brightest part of the western wall of a cavity that contains the $\mathrm{HH} 34$ jet. Re 

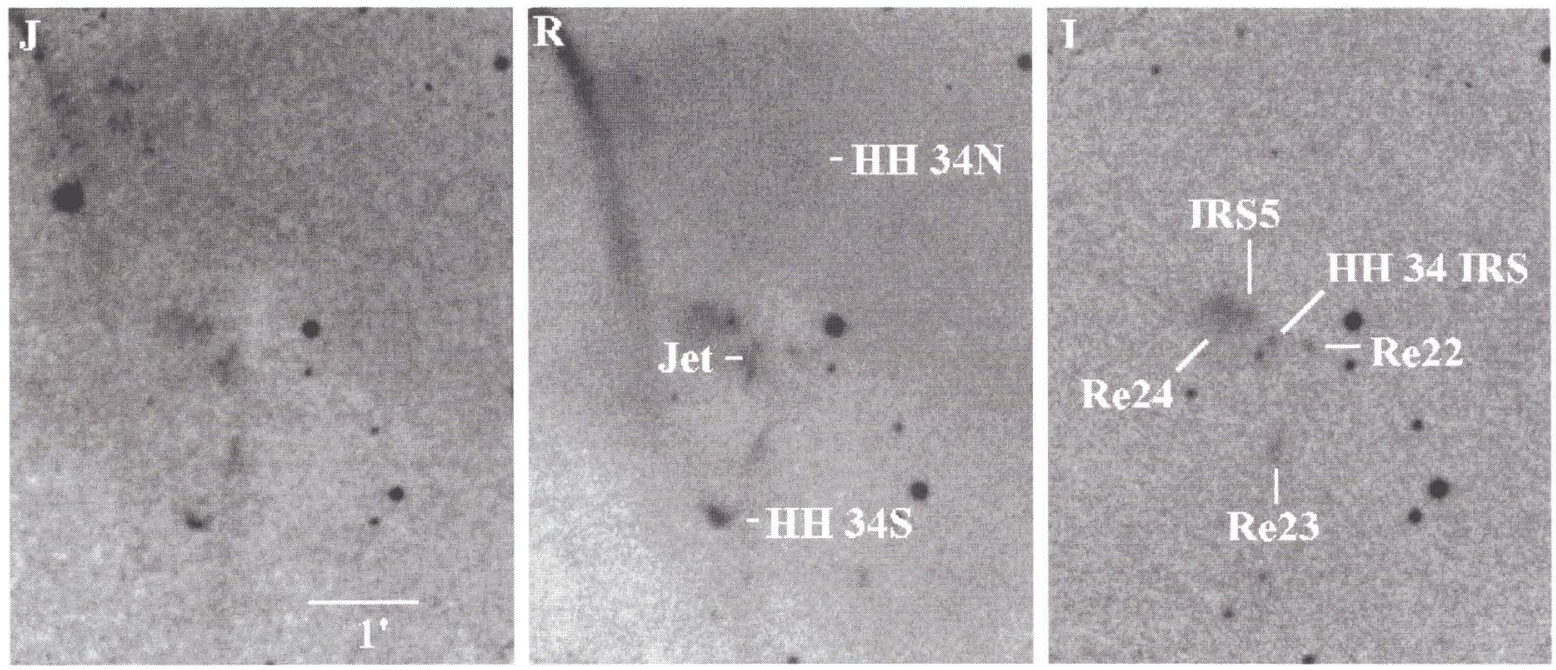

Figure 1-ESO/SERC IIIaJ (J), IIIaF (R) and IVN (I) images of the HH 34 system. The reflection nebulosity Re 23 may be tracing a cavity created by the HH 34 jet. The location of the energy source (HH 34 IRS) is clearly seen in the IVN band. North is up and east is left in all images.

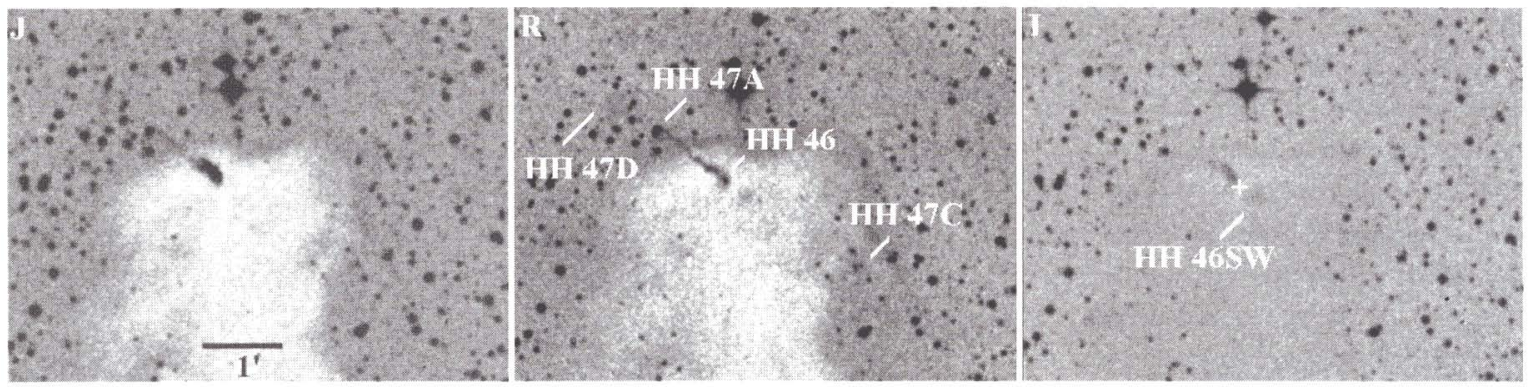

Figure 2-ESO/SERC IIIaJ (J), IIIaF (R) and IVN (I) images of the HH $46 / 47$ system. The position of HH $46 / 47$

IRS is indicated by the cross.

23 shows a different morphology in the IIIaJ and IIIaF images and has been associated with a linear $\mathrm{H}_{2}$ emission structure which is possibly a separate outflow (Zealey, Suters \& Randall 1993). HH 34N is just visible in the IIIaF band, and no visible counterparts to an arc of infrared emission north of $\mathrm{HH} 34$ IRS are seen.

\section{2 $\mathrm{HH} 46 / 47$}

$\mathrm{HH} 46 / 47$ is also a well studied flow at visible, infrared and millimetre wavelengths. Many of the optical features discovered using CCD techniques are clearly visible on the ESO/SERC survey films, without enhancement.

The IIIaJ and IIIaF images (Figure 2) of $\mathrm{HH}$ $46 / 47$ show the faint emission features associated with the blue-shifted, bow-shaped nebulosity $\mathrm{HH}$ $47 \mathrm{D}$ and red-shifted bow-shock working surface $\mathrm{HH}$ $47 \mathrm{C}$ as observed in the [S II] CCD images of Eislöffel \& Mundt (1994).

The strong reflection nebulosity around the source and in the region of $\mathrm{HH} 46$ dominates the IIIaJ image. Both the IVN (Figure 2) image and the infrared image (Figure 3; Zealey et al. 1993) fail to show a central point source, due to the presence of a dense obscuring disk surrounding the source.
The IVN image displays emission, which may be due to reflection nebulosity associated with $\mathrm{HH} 46$, $\mathrm{HH} 46 \mathrm{SW}$ and the region of the counter-jet.

None of the IVN, IIIaF or IIIaJ images shows emission from the red-shifted flow which is clearly seen in the $2 \cdot 12 \mu \mathrm{m}$ image (Zealey et al. 1993). This again indicates the high obscuration in this direction due to the dark cloud in which $\mathrm{HH} 46 / 47$ is embedded.

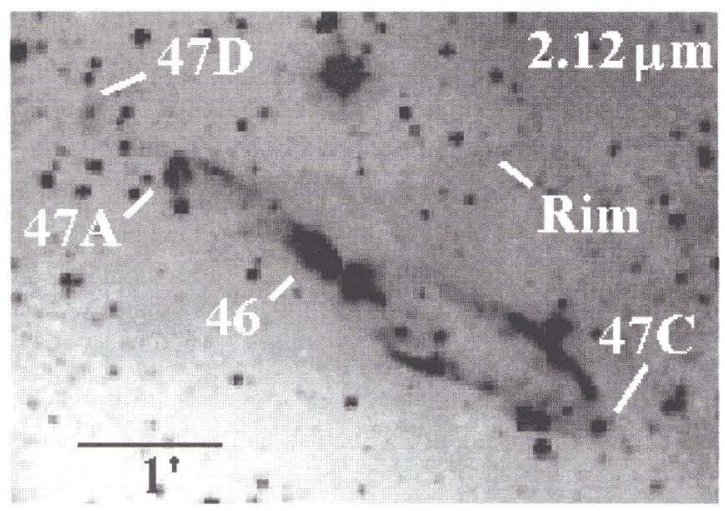

Figure 3- $\mathrm{H}_{2}$ image of the $\mathrm{HH} 46 / 47$ system from Zealey et al. (1993). The blue-shifted flow terminates in HH 47D. The red-shifted lobe occupying the lower right of the image can be traced back to HH 46/47 IRS, at field centre. 


\section{Future Developments}

This project has shown the effectiveness of lowcost video digitising in analysing and searching ESO/SERC Sky Surveys for emission line sources. Future surveys, including the proposed $\mathrm{H} \alpha$ emission line survey of the southern sky to be carried out by the UKST, will provide deep, high-resolution images of Galactic plane sources.

The application of digital techniques to archival photographic material, while not providing the depth of CCD imaging, does allow the identification of the main features associated with outflows. It is particularly applicable to the discovery of new structures associated with flows more extensive than can be covered by existing CCD formats $\left(<10^{\prime}\right)$.

Ongoing work on the detection of filamentary sources associated with supernova remnants, wind shells and star formation regions is difficult near strong reflection or emission nebulosity. The use of background subtraction techniques using low-cost digital imaging systems will increase our success in detecting such features.

We intend making the final image database available on the World Wide Web (WWW) with the hope that future work at near-infrared wavelengths will determine whether reddened sources visible on the IVN images are driving the optical $\mathrm{HH}$ flows seen in the IIIaJ and IIIaF images. Access to online catalogues has grown substantially over the last few years. An advantage of putting imaging or large data catalogues on the Internet is that the data are easily accessible and available in the public domain as soon as refereeing is completed.

Examples of catalogues relevant to star formation include Bo Reipurth's A General Catalogue of Herbig-
Haro objects (Reipurth 1994) and the Catalogue of Emission Line Stars (Herbig \& Bell 1988).

\section{Acknowledgments}

The preparation of this catalogue would not have been possible without the dedication of the many individuals who worked on the ESO/SERC Sky Surveys. Particular thanks are due to the staff of the AAO UK Schmidt Telescope.

Eislöffel, J., \& Mundt, R. 1994, A\&A, 284, 530

Hartigan, P., \& Lada, C. J. 1985, ApJS, 59, 383

Herbig, G. H. 1972, Lick Observatory Bulletin No. 658

Herbig, G. H., \& Bell, K. R. 1988, Lick Observatory Bulletin No. 1111

Malin, D. F., Ogura, K., \& Walsh, J. R. 1987, MNRAS, 227,361

Mundt, R. 1988, in Formation and Evolution of Low Mass Stars, NATO ASI vol. 241, ed. A. K. Dupree \& M. T. V. Lago (Dordrecht: Kluwer), 257

Reipurth, B. 1985, ApJS, 61, 319

Reipurth, B. 1994, A General Catalogue of HerbigHaro objects, electronically published via anon. ftp to ftp.hq.eso.org, directory/pub/Catalogs/Herbig-Haro

Schwartz, R. D. 1977, ApJS, 35, 161

Strom, K. M., Strom, S. E., Wolff, S. C., Morgan, J., \& Wenz, M. 1986, ApJS, 62, 39

Von Hippel, T., Burnell, S. J., \& Williams, P. M. 1988, A\&A, 74, 431

Zealey, W. J., Moore, G. K. G., Ihnat, P., \& Suters, M. G. 1994, Working Group on Wide-field Imaging, IAU Commission 9, Newsletter 6

Zealey, W. J., Suters, M., \& Randall, P. 1993, PASP, 10, 203

Zealey, W. J., Williams, P. M., Sandell, G., Taylor, K. N. R., \& Ray, T. P. 1992, A\&A, 262, 570 